\title{
OTOMOMICS
}

Revista de economía, empresa y sociedad

Dosier «Vectores de sostenibilidad: visiones desde la economía» Coordinador: Albert Puig Gómez

METODOLOGÍA DE MEDIDA

\section{Pobrezas multidimensionales}

\section{Agustí Pérez-Foguet}

Universitat Politècnica de Catalunya

RESUMEN En los últimos veinte años se ha consolidado una metodología que nos permite medir, con un alto grado de consenso internacional, la pobreza multidimensional. Disponer de una metodología de medida ha facilitado concretar y operativizar el concepto. En paralelo, se han propuesto y consolidado propuestas de pobrezas «sectoriales», con entidad conceptual propia. El vínculo entre las dimensiones de la pobreza multidimensional y las pobrezas sectoriales es diverso. En este artículo, se discuten distintos enfoques (pobreza laboral, energética, hídrica, de saneamiento y menstrual). Se argumenta que reducir las pobrezas sectoriales a dimensiones de la pobreza multidimensional limita su utilidad e impacto. Se propone avanzar en la caracterización multidimensional de dichas pobrezas sectoriales, aprovechando para ello el trabajo metodológico realizado previamente en términos de la pobreza multidimensional, en singular, ya consolidado.

PALABRAS CLAVE índice de pobreza multidimensional; asequibilidad; pobreza energética; pobreza hídrica; escalera de nivel de servicio; derechos humanos

\section{MEASUREMENT METHODOLOGY}

\section{Multidimensional poverties}

\begin{abstract}
In the last twenty years, a methodology that allows us to measure, with a high degree of international consensus, multidimensional poverty has been consolidated. The definition of the measurement methodology has facilitated to specify and put into operation the concept. In parallel, "sectoral" poverty proposals have been used and finally consolidated, with their own conceptual importance and usefulness. The link between the dimensions of multidimensional poverty and sectoral poverties is diverse. This paper briefly presents and discusses different approaches (labour, energy, water, sanitation, and period poverties). It is argued that reducing sectoral poverties to the dimensions of multidimensional poverty limits their usefulness and impact. The methodological work done previously in terms of measurement of multidimensional poverty, in the singular, helps also to advance in the multidimensional characterization of said sectoral poverties.
\end{abstract}

KEYWORDS multimensional poverty index; affordability; energy poverty; water poverty; service level ladder; human rights 


\section{Introducción}

Mucho se ha escrito sobre pobreza, y tal como muestran Atkinson (1987), Feres y Mancero (2001a), desde hace tiempo. Tanto en lengua catalana ${ }^{1}$ como española ${ }^{2}$ se define pobreza con las acepciones de falta, escasez, insuficiencia; también, en español, escaso haber de la gente pobre; en catalán, falta o limitación en lo necesario para vivir; y en ambos, cualidad de pobre; siendo pobre, como sustantivo, necesitado, que no tiene lo necesario para vivir; mendigo (persona que habitualmente pide limosna). Como adjetivo, también en ambas lenguas, escaso, insuficiente; en castellano, además, humilde, de poco valor o entidad; y en catalán, ineficiente y también, antepuesto al nombre, indica la conmiseración, el desdén. El uso en inglés del término pobreza (poverty) ${ }^{3}$ es coincidente, aunque aporta matices, destacando: "deficiencia en las propiedades necesarias o cualidades deseables, especificadas", y "no adecuado"; respecto a pobre (poor), su uso es casi exclusivo como adjetivo, excepto en «los pobres» (the poor), donde se refiere al colectivo de personas que no disponen de recursos para una calidad de vida razonable (el singular se denota como «persona pobre»).

Es habitual caracterizar a la persona (familia) pobre por alguna magnitud económica, tipo renta per cápita (o familiar). La lógica subyacente es que si se dispone de dinero suficiente se puede conseguir mejorar la calidad de vida al nivel deseado. Quizás no se puede determinar de forma precisa el nivel de «lo necesario para vivir» o la "calidad de vida razonable» (menos aún si las comparaciones son de la humanidad globalmente), pero en contextos equiparables los que disponen de más dinero tienen más posibilidades de disfrutar de una calidad de vida deseable, valoradas por ellos mismos de más calidad. La división entre ser o no ser pobre puede ser difusa y difícil de caracterizar objetivamente, pero cuando se pone rostro a posiciones extremas las diferencias son evidentes. Dada la dificultad de comparar en términos absolutos magnitudes tipo renta entre contextos muy distintos, suelen armonizarse internacionalmente y/o ser consideradas en valor relativo. Las comparaciones no son solo entre países; es habitual comparar rentas entre regiones de los Estados, y hasta de barrios de una misma ciudad. Se transmite de forma muy directa la percepción de que, geográfica y administrativamente, la renta (y, por tanto, la posibilidad de pobreza) no se distribuye de forma uniforme, sino que comparte características que van más allá del individuo o la familia.

Pese a ser una primera aproximación, los valores de renta media (u otras medidas de las distribuciones) en conjuntos poblacionales no son propiamente indicadores de pobreza. De acuerdo con Sen (1976), la medición de la pobreza consta de dos etapas: La identificación de quienes son pobres y quienes no, y la agregación de la cantidad de pobreza que tienen dichos pobres. La identificación exige la definición de un umbral sobre la cantidad que se utiliza para caracterizarla, valor que se conoce como línea de pobreza, y que recoge la problemática antes indicada sobre ser o no ser pobre. La agregación exige disponer de una magnitud agregable poblacionalmente, por ejemplo, la diferencia entre el valor de la línea de pobreza en renta y la renta real de cada uno de los pobres. Un indicador clásico es el utilizado actualmente para el seguimiento del objetivo de desarrollo sostenible (ODS) 1.1, la erradicación de la pobreza extrema (UN, 2021): personas con renta menor a 1,25 USD PPA (línea de pobreza extrema internacionalmente aceptada).

\section{La multidimensionalidad}

La idea de disponer de un único indicador para caracterizar las situaciones que identificamos como pobreza es claramente limitada. Sen (1990) argumenta que los medios, los recursos, no pueden ser la única base informacional que sustente la justicia, dada la diversidad interpersonal en la conversión de bienes en libertades reales. De igual modo sucede con pobreza: se puede argumentar que la disponibilidad de renta no nos da información suficiente para caracterizar las privaciones que realmente sufre una persona y que nos hacen juzgarla (clasificarla) como pobre. Desde un punto de vista más práctico, si definimos una forma de medir (indicador) para priorizar o seguir políticas, pero cuando la aplicamos nos sale igual (de mal) en todos los casos, es evidente que el indicador deja de ser útil. Si en algún lugar la mayoría son pobres (e igual de pobres, entre 0 y 1,25 USD, no es mucha diferencia), ¿para qué nos es útil la informa-

1. https://dlc.iec.cat/ [fecha de consulta: septiembre de 2021].

2. https://dle.rae.es/ [fecha de consulta: septiembre de 2021].

3. https://www.collinsdictionary.com/dictionary/english [fecha de consulta: septiembre de 2021]. 
ción? (iy el esfuerzo de recogerla!). Los indicadores de distribución de renta tienen distribuciones tan, tan asimétricas, y la conversión de dinero en libertad de elección es tan diversa, que el enfoque clásico de renta bajo línea de pobreza deja de ser útil más allá de contextos parecidos, relativamente uniformes.

La necesidad de pasar de un indicador de pobreza «unidimensional» a otro «multidimensional», con esta terminología, se remonta a los trabajos de Bourguignon y Chakravarty (2003), Tsui (2002) y Alkire y Foster (2007, 2011). El índice de pobreza multidimensional (MPI) presentado por Alkire y Santos (2014) mide un conjunto de diez privaciones en tres dimensiones principales: salud, educación y calidad de vida. Ha tenido amplia proyección en el ámbito global al ser integrado en la agenda internacional por el Programa de Naciones Unidas para el Desarrollo (PNUD) en sus informes de seguimiento anual..$^{4} \mathrm{~L}$ identificación de las personas pobres se realiza comparando el peso de las subdimensiones en las que hay privación con el umbral del 33 \% (equivalente a que una de las tres dimensiones principales no llegue a los umbrales mínimos definidos); y las personas en pobreza severa con el umbral 50 \% (equivalente a una y media de las tres).

Es ilustrativo comparar la propuesta de variables incluidas en el MPI con las variables de las categorías de necesidad básicas presentadas por Feres y Mancero (2001b), sobre trabajos previos en América Latina en la década de 1980. Cambian el enfoque y el método, pero no tanto las dimensiones. En el MPI, la dimensión de educación se mantiene, la de estándares de vida (hogar, agua y saneamiento) se complementa con dos indicadores vinculados a la energía (el consumo de combustibles fósiles para cocinar y no disponer de electricidad en el hogar), se introducen indicadores de salud y no se incluyen los de capacidad económica en el hogar. Por otro lado, en el contexto de Europa, es destacable también la conexión entre el MPI y el «Estado de privación material y social severa» del indicador AROPE (indicador de Riesgo de pobreza y/o exclusión social, recientemente actualizado en el Plan de Acción del Pilar Europeo de Derechos Sociales 2030). ${ }^{5}$ La identificación de las personas en estado de privación material y social severa se basa en la falta de 7 o más de un listado de 13 posibles privaciones, 7 a nivel de hogar y 6 individuales. ${ }^{6}$

Un requisito para poder medir la pobreza multidimensional es disponer de estimaciones de las probabilidades de distribución conjunta de las variables implicadas. No es suficiente con las distribuciones marginales. Esto no es solo un detalle técnico, sino que supone un paso adelante en términos de conocer realmente la pobreza de las personas, tanto en el sentido de cantidad de pobres como en la intensidad de la pobreza a la que están sometidos. Remarcablemente, los indicadores de seguimiento del ODS 1.2 incluyen el porcentaje de personas bajo línea de pobreza nacional (entendida en términos económicos), y también de proporción de personas que viven en situación de pobreza en todas sus dimensiones según los estándares nacionales. Se abre, por tanto, la caracterización de pobreza a múltiples dimensiones, con la posibilidad de ser redefinida localmente.

\section{Las pobrezas}

La segunda idea de la reflexión me lleva a ampliar el objeto de análisis: de la pobreza pasaré a las «pobrezas». No es una transición directa, ni uniforme. El plural «pobrezas» indica que pueden existir varias, que tendrán cualidades particulares, pero obedecerán a la idea de semántica de pobreza ya presentada: escaso, humilde, de poco valor, que no da sustento; con el matiz en inglés de inadecuado. Para entender estas pobrezas, digamos con apellido, es necesario referirse a su contexto, origen y usos. A continuación, introduciré algunas de ellas.

La primera pobreza que introduzco es la «pobreza laboral». Presenta diversas interpretaciones y problemas técnicos para su definición precisa y medición (De la Cal, 2014), pero a grandes rasgos se entiende como el porcentaje de población que, aunque dispone de trabajo remunerado, tiene rentas (familiares) por debajo de una determinada línea de pobreza. La modulación del nombre en realidad hace referencia a una segmentación de la población de estudio (trabajan/no trabajan), junto con la aplicación estándar de medidas unidimensionales de la pobreza (renta familiar por debajo o no del umbral). El análisis internacional comparado muestra que pueden identificarse diversos factores en la realidad

4. http://hdr.undp.org/en/2020-MPI [fecha de consulta: septiembre de 2021].

5. https://eur-lex.europa.eu/legal-content/ES/TXT/?uri=COM:2021:102:FIN [fecha de consulta: septiembre de 2021].

6. https://ec.europa.eu/eurostat/statistics-explained/index.php?title=Glossary:Severe_material_and_social_deprivation_rate_ (SMSD) [fecha de consulta: septiembre de 2021]. 
de este tipo de pobreza: salarios individuales de los miembros del hogar, otros ingresos y su tipología, estructura del hogar y ciclo vital de sus miembros (De la Cal, 2014), pero no parece que haya sido necesario afinar más el concepto, y se presenta como subsidiario de la definición de pobreza general adoptada.

Otra pobreza, lamentablemente conocida, es la «pobreza energética», 7 que ha ido cobrando protagonismo público los últimos años. En España el concepto ya tenía recorrido, pero tras el verano de 2021 ha pasado a ser titular durante días consecutivos. Las subidas extraordinarias del precio de la energía eléctrica ya han afectado a los hogares, y, de forma que aún está por ver, afectarán a la economía en su conjunto (el futuro lector podrá contrastar qué es lo que realmente sucedió y qué impacto diferencial se produjo en la población de menor renta). Una hipótesis razonable es que las personas «no pobres energéticamente» (hasta el momento), que nunca habían sufrido por pagar servicios energéticos, pueden ver incrementados muy significativamente los costes asociados, y eso puede poner en jaque el confort de los hogares en invierno. Situación que, de hecho, parte de la población ya conoce (los que antes ya han sufrido la llamada «pobreza energética").

Según el Observatorio de Pobreza Energética de la Comisión Europea, ${ }^{8}$ la falta de capacidad de pago es crítica, pero no solo de servicios esenciales de energía, sino también de los asociados a las características de la vivienda y edificios (y su adecuación y/o renovación para mantener el confort y aumentar la eficiencia). También queda claro que es una situación que afecta a un porcentaje elevado de los hogares de la Unión («más de 50 millones de hogares en la Unión Europea»). Se es pobre energéticamente o no, por una cuestión de acceso a servicios y bienes, cosa que se estructura en torno a la capacidad de pago por estos servicios. Esto, que en primera aproximación parece adecuado, deja a las personas sin capacidad efectiva de maniobra ante las subidas exponenciales de precios. No me extenderé más, pero quizás dimensiones como los niveles de autoconsumo, la pertenencia a un servicio de suministro por comunidades energéticas locales, la disposición de legislaciones «favorables a», los servicios de "asistencia técnica para", etc., podrían ser elementos complementarios. Entender más multidimensionalmente la pobreza energética abre la puerta para salir de una visión reactiva (no podemos pagar lo que nos ofrecen otros) y entrar en otra propositiva (sin estos medios y capacidades no podemos cubrir nuestras necesidades mínimas). No pretendo menospreciar el problema real de pobreza económica subyacente, pero sí enfatizar la trampa de vincular la pobreza energética solo con la disponibilidad de dinero.

A diferencia de la pobreza energética, la «pobreza hídrica» (relativa al agua) es mayoritariamente utilizada en términos distintos del pago por bienes y servicios. El concepto nace en el campo de la gestión y las políticas ambientales y de desarrollo, y se articula y justifica en Sullivan (2002), trabajo en el que presenta la propuesta de construir una valoración integrada del estrés hídrico y la escasez de agua, que vincule estimaciones de parámetros físicos de disponibilidad del agua con variables socioeconómicas que reflejen pobreza. Sullivan et al. (2003) concretan: «Las dimensiones del índice deben incluir medidas de: Acceso al agua; Cantidad, calidad y variabilidad del agua; Usos del agua (doméstico, alimentación y procesos productivos); Capacidad para la gestión de recursos hídricos y Aspectos ambientales». La propuesta de índice se realiza con vocación de ser aplicada por «decisores, y al mismo tiempo facilite el empoderamiento de los pobres para su participación activa en la identificación de mejores intervenciones en el sector, y en general de los presupuestos de desarrollo».

Si bien el planteamiento inicial se focalizó en el hogar, las aplicaciones se han desarrollado mayoritariamente a escalas comunitarias y geográficamente mayores (Lawrence et al., 2002; Cullis y Regan, 2004; Sullivan y Meigh, 2007). Entre los trabajos realizados que han supuesto un avance de la propuesta, destaco el de Giné-Garriga y Pérez-Foguet (2010), que proponen mejoras en la selección y agregación de información. Pérez-Foguet y Giné-Garriga (2011) ofrecen una interpretación combinada con el enfoque Presión - Estado - Respuesta en cuencas hidrográficas, y Giné-Garriga y Pérez-Foguet (2013), su particularización y diferenciación desde la perspectiva del abastecimiento de agua, saneamiento e higiene.

La propuesta de índice de pobreza hídrica supuso un avance respecto del uso habitual de indicadores de cobertura de infraestructura, el habitual en contextos de desarrollo, pero no se estableció como la unidad nueva de medida en términos de seguimiento de inversiones. Por un lado, no es sencillo disponer de la información necesaria para un uso

7. https://ec.europa.eu/energy/topics/markets-and-consumers/energy-consumer-rights/energy-poverty_en [fecha de consulta: septiembre de 2021].

8. https://www.energypoverty.eu/about/what-energy-poverty [fecha de consulta: septiembre de 2021]. 
individualizado (persona, hogar) del marco conceptual planteado, pero, por otro, desde la lógica de las relaciones de pobreza y agua, es extraño pensar que el problema del agua es básicamente personal o del hogar. La componente de acceso individual tiene un peso pequeño respecto a la del medio físico -ambiental- social en el que existimos. La relación entre agua y pobreza está muy ligada al contexto local concreto; el último paso, la individualización del servicio de agua urbana en el hogar, es una parte pequeña del «problema» del acceso al agua potable.

Con la declaración del derecho humano al agua y al saneamiento, ${ }^{9}$ se complementa el enfoque previo con un especial énfasis en el individuo, el titular de derechos. El marco de los derechos humanos ofrece una descripción alternativa de dimensiones para el análisis de cómo se cubren las necesidades mínimas. Se definen unas componentes normativas, específicas de este derecho particular, y otras transversales. Destaco, entre las normativas que se aplican a los servicios de agua potable y saneamiento, la asequibilidad (estableciendo la recomendación de un coste máximo de los servicios asociados del $3 \%$ de la renta del hogar).

Giné-Garriga y Pérez-Foguet (2019) presentan la aplicación de la metodología de Alkire y Foster para medir la «pobreza de saneamiento» desde un enfoque de realización progresiva del derecho humano e incluyendo sus cinco dimensiones normativas. La propuesta se puede aplicar a otros conceptos multidimensionales definidos mediante gradaciones de niveles de servicio ("escaleras" en terminología del Programa Conjunto PNUD/OMS que coordina y sigue los niveles de servicio estandarizados en agua, saneamiento e higiene a nivel internacional)..$^{10}$ Los indicadores utilizados actualmente para el seguimiento de los ODS 6.1, alcanzar acceso universal y equitativo al agua potable, y 6.2, saneamiento e higiene adecuados, equitativos y universales (finalizando con la defecación al aire libre y con especial atención a las necesidades de mujeres, niñas y aquellos en situaciones vulnerables), se definen ambos en términos de porcentaje de población que accede a un nivel de servicio (UN, 2021) y se refieren a un derecho humano con dimensiones normativas; por tanto, ambos se pueden medir desde esta perspectiva. El resultado será, en cualquier aplicación, distinto al obtenido en Giné-Garriga y Pérez-Foguet (2013), basado en los planteamientos previos de "pobreza hídrica", no solo por método, sino por la interpretación del concepto medido.

Otra de las aportaciones del enfoque de derechos, en la que no me extenderé, hace referencia a los titulares de obligaciones, los Estados. Destaca la conexión directa con el concepto de pobreza hídrica: la disponibilidad del recurso y el acceso a este por parte de la población. Garantizar que el agua y el saneamiento son un derecho humano ayuda a blindar este uso por encima de otros cuando entren en competencia. Obliga a seguir la situación específicamente de grupos sociales y situaciones de dependencia y vulnerabilidad (Ezbakhe et al., 2019). Si no hay agua, no hay vida; lo primero que tiene que asegurar el agua es la vida (en torno al $60 \%$ de nuestra masa corporal es agua, y las moléculas se renuevan en media cada diez días). Si estamos vivos, seremos pobres hídricos, o no, todo dependerá de que seamos capaces de gestionar los recursos hídricos disponibles, mantenerlos accesibles y de calidad, priorizando los usos que consideremos necesarios para nuestro desarrollo. Que el sujeto de la frase previa deba ser el hogar, o la comunidad, ciudad, cuenca hidrográfica, Estado..., o todas ellas, es otro aspecto sobre el que reflexionar, pero el titular de obligaciones (en el ámbito internacional) es el que debe respetar, proteger y cumplir. ${ }^{11}$

Por último, destaco el uso del concepto «pobreza menstrual». El concepto, a nivel internacional, ${ }^{12}$ parte de que si no hay acceso a ciertas soluciones higiénicas, no pueden usarse; si no se conocen, no pueden usarse; pero además se resalta que si socialmente se penaliza o estigmatiza a la persona que menstrúa, es más difícil de gestionar y tiene efectos negativos en otras esferas (salud, escolarización, participación comunitaria, etc.). Que el coste sea proporcionalmente elevado sobre la renta es crítico, véanse los casos recientes de Nueva Zelanda o Escocia (elDiario.es, 3/6/2020), pero van más allá, "es una cuestión social y política» (UNFPA, 2021), por lo que un enfoque exclusivamente económico no es suficiente para abordarlo.

Recientemente, en el Parlamento Vasco se presentó y aprobó la proposición no de ley relativa al impulso de políticas públicas que aborden la pobreza menstrual, ${ }^{13}$ que, partiendo del problema del coste, en especial para las personas pobres o vulnerables, insta a que se realice un estudio específico sobre la salud e higiene menstrual desde una perspectiva integral e interseccional. En el contexto de la reflexión de este artículo diría, «y caracterizado multidimensionalmente», no

9. Resolución 64/292, Asamblea General de las Naciones Unidas (2010).

10. https://washdata.org/monitoring [fecha de consulta: septiembre de 2021].

11. https://www.ohchr.org/sp/issues/escr/pages/whataretheobligationsofstatesonescr.aspx [fecha de consulta: septiembre de 2021].

12. https://www.actionaid.org.uk/our-work/womens-rights/period-poverty [fecha de consulta: septiembre de 2021].

13. https://www.irekia.euskadi.eus/es/events/69416-pleno-ordinario [fecha de consulta: septiembre de 2021]. 
solo por indicadores económicos relativos al acceso a los productos higiénicos, sino, quizás, con dimensiones relativas a la presencia y características de aseos en hogar, escuelas, centros de trabajo y espacio público, conocimiento previo, formación y provisión de alternativas a parte de niñas y adolescentes, etc. También recientemente, en el Programa Conjunto PNUD/OMS, y bajo el enfoque de «salud menstrual», ${ }^{14}$ se profundiza en la definición del concepto y en propuestas de indicadores que permitan seguir la evolución internacional al respecto. ${ }^{15}$ Es de prever que en los próximos años se consolide una propuesta de escalera relativa a las condiciones de «salud menstrual», al mismo nivel que se diferencian y definen las de «higiene», «saneamiento» y «agua para el consumo humano».

\section{Reflexiones finales}

Interpretar las distintas pobrezas como las dimensiones de la «pobreza», digamos que "completa», de las personas puede enmascarar con facilidad características propias que cada concepto de pobreza específica ha querido destacar. Interpretar las distintas pobrezas como un problema de no poder convertir dinero en bienes y servicios para disponer de un mínimo de calidad de vida restringe el análisis finalmente a una única dimensión, situación precisamente que se pretende superar. Que algo sea fácil (unidimensional) es positivo, da una primera aproximación a la realidad y puede ser útil para comparar situaciones parecidas; pero que algo sea un poco menos fácil (pasemos de una a tres o cuatro dimensiones...) a cambio de entender mejor la hipercompleja realidad nos lo deberíamos poder permitir. Definir y reconocer en las pobrezas componentes, dimensiones y resortes que las constituyen más allá del dinero facilita plantearse opciones alternativas a las dominantes (formuladas mayoritariamente desde la unidimensionalidad).

\section{Referencias bibliográficas}

ATKINSON, A. B. (1987, julio). «On the Measurement of Poverty». En: Econometrica, vol. 55, núm. 4, págs. 749-764 [en línea]. Disponible en: https://www.ophi.org.uk/wp-content/uploads/Atkinson-1987.pdf [fecha de consulta: septiembre 2021].

ALKIRE, S.; FOSTER, J. (2007). «Counting and multidimensional poverty measurement». En: OPHI Working Paper (revisado en 2008), vol. 7. Oxford: Universidad de Oxford.

ALKIRE, S.; FOSTER, J. (2011). "Counting and multidimensional poverty measurement». En: Journal of Public Economics, vol. 95, núm. 448, págs, 476-487 [en línea]. DOl: https://doi.org/10.1016/j.jpubeco.2010.11.006

ALKIRE, S.; SANTOS, M. E. (2014). «Measuring Acute Poverty in the Developing World: Robustness and Scope of the Multidimensional Poverty Index». En: World Development, vol. 59, núm. 455, págs. 251-274 [en línea]. DOI: https:// doi.org/10.1016/j.worlddev.2014.01.026

ALKIRE, S.; KANAGARATNAM, U.; SUPPA, N. (2020). «The global Multidimensional Poverty Index (MPI) 2020». En: OPHI MPI Methodological Note, vol. 49, núm. 2 [en línea]. Oxford: Oxford Poverty and Human Development Initiative, University of Oxford. DOl: https://doi.org/10.1080/13600818.2020.1854209

BOURGUIGNON, F.; CHAKRAVARTY, S. (2003). «The measurement of multidimensional poverty». En: The Journal of Economy Inequal, vol. 1, págs. 25-49 [en línea]. DOl: https://doi.org/10.1023/A:1023913831342

CULLIS, J.; O'REGAN, D. (2004). «Targeting the water-poor through water poverty mapping». En: Water Policy, núm. 6, págs. 397-411 [en línea]. DOI: https://doi.org/10.2166/wp.2004.0026

14. https://www.ncbi.nlm.nih.gov/pmc/articles/PMC8098749/pdf/ZRHM_29_1911618.pdf [fecha de consulta: septiembre de 2021].

15. https://washdata.org/monitoring/menstrual-health [fecha de consulta: septiembre de 2021]. 
DE LA CAL, M. L. (2014). «Tema Central: La Pobreza Laboral». En: Boletín de recursos de información, núm. 40 [en línea]. UPV/EHU: Centro de Documentación Hegoa. Disponible en: https://boletin.hegoa.ehu.eus/mail/33 [fecha de consulta: septiembre de 2021].

elDiario.es (2020, junio). «Nueva Zelanda aborda la "pobreza menstrual” con tampones y compresas gratuitos para las estudiantes de secundaria».

EZBAKHE, F.; GINÉ-GARRIGA. R.; PÉREZ-FOGUET, A. (2019). «Leaving no one behind: Evaluating access to water, sanitation and hygiene for vulnerable and marginalized groups». En: Science of The Total Environment, núm. 683, págs. 537-546 [en línea]. DOI: https://doi.org/10.1016/j.scitotenv.2019.05.207

FERES, J. C.; MANCERO, X. (2001a). «Enfoques para la medición de la pobreza: breve revisión de la literatura». En: Serie Estudios Estadísticos y Prospectivos. LC/L.1479-P, CEPAL. ISBN: 9213217064.

FERES, J. C.; MANCERO, X. (2001b). «El método de las necesidades básicas insatisfechas (NBI) y sus aplicaciones a América Latina». En: Serie Estudios Estadísticos y Prospectivos. LC/L.1491-P, CEPAL. ISBN: 9213217919.

GINÉ-GARRIGA, R.; PÉREZ-FOGUET, A. (2010.). «Improved Method to Calculate a Water Poverty Index at Local Scale». En: Journal Environmental Engineering, núm. 136, págs. 1287-1298 [en línea]. DOl: https://doi.org/10.1061/ (ASCE)EE.1943-7870.0000255

GINÉ-GARRIGA, R.; PÉREZ-FOGUET, A. (2013). «Unravelling the Linkages Between Water, Sanitation, Hygiene and Rural Poverty: The WASH Poverty Index». En: Water Resources Management, núm. 27, págs. 1501-1515 [en línea]. DOI: https://doi.org/10.1007/s11269-012-0251-6

GINÉ-GARRIGA, R.; PÉREZ-FOGUET, A. (2019). «Monitoring and targeting the sanitation poor: A multidimensional approach». En: Natural Resources Forum, vol. 43, núm. 2, págs. 82-94 [en línea]. DOl: https://doi.org/10.1111/14778947.12171

LAWRENCE, P.; MEIGH, J.; SULLIVAN C. (2002). «The Water Poverty Index: an International Comparison». En: Keele Economics Research Papers, núm. 19.

PÉREZ-FOGUET, A.; GINÉ-GARRIGA, R. (2011). «Analyzing Water Poverty in Basins». En: Water Resources Management, núm. 25, pág. 3595 [en línea]. DOl: https://doi.org/10.1007/s11269-011-9872-4

SEN, A. (1976). «Poverty: An Ordinal Approach to Measurement». En: Econometrica, vol. 44, núm. 2, págs. 219-231 [en línea]. DOl: https://doi.org/10.2307/1912718

SEN, A. (1990). «Justice: Means versus Freedoms». En: Philosophy \& Public Affairs, vol. 19, núm. 2, págs. $111-121$ [en línea]. Disponible en: https://www.jstor.org/stable/2265406

SULLIVAN, C. (2002). "Calculating a water Poverty Index». En: World Development, vol. 30, núm. 7, págs. 1195-1210 [en línea]. DOI: https://doi.org/10.1016/S0305-750X(02)00035-9

SULLIVAN. C. A.; MEIGH, J. (2007). «Integration of the biophysical and social sciences using an indicator approach: addressing water problems at different scales». En: Water Resources Management, vol. 21, núm. 1, págs. 111-128 [en línea]. DOI: https://doi.org/10.1007/s11269-006-9044-0

SULLIVAN, C. A. et al. (2003). "The Water Poverty Index: Development and application at the community scale». En: Natural Resources Forum, núm. 27, págs. 189-199 [en línea]. DOI: https://doi.org/10.1111/1477-8947.00054

TSUI, K. Y. (2002). «Multidimensional poverty indices». En: Social Choice and Welfare, núm. 19, págs. 69-93 [en línea]. DOI: https://doi.org/10.1007/s355-002-8326-3

UN (2021). «SDG Indicators: Global indicator framework for the Sustainable Development Goals and targets of the 2030 Agenda for Sustainable Development». En: Sustainable Development Goals [en línea]. Disponible en: https://unstats. un.org/sdgs/indicators/indicators-list/ [fecha de consulta: septiembre de 2021].

UNFPA (2021). «Menstruation and human rights - Frequently asked questions». En: United Nations Population Fund [en línea]. Disponible en: https://www.unfpa.org/menstruationfaq [fecha de consulta: septiembre de 2021]. 
Cita recomendada: PÉREZ-FOGUET, Agustí. Pobrezas multidimensionales. Oikonomics [en línea]. Noviembre 2021, n. 16. ISSN: 2339-9546. DOI: https://doi.org/10.7238/o.n16.2109

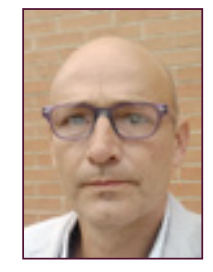

\section{Agustí Pérez-Foguet}

agusti.perez@upc.edu

\section{Universitat Politècnica de Catalunya}

Dr. Ingeniero de Caminos, Canales y Puertos. Catedrático de universidad, ámbito Ingeniería y Arquitectura, área de conocimiento Matemática Aplicada. Investigador de la Red de Excelencia en Investigación sobre la pobreza hídrica WAPONET (CSO2017-90702-REDT), Ministerio de Economía, Industria y Competitividad, Gobierno de España.

Los textos publicados en esta revista están sujetos -si no se indica lo contrario- a una licencia de Reconocimiento 4.0 Internacional de Creative Commons. Puede copiarlos, distribuirlos, comunicarlos públicamente, hacer obras derivadas siempre que reconozca los créditos de las obras (autoría, nombre de la revista, institución editora) de la manera especificada por los autores o por la revista. La licencia completa se puede consultar en https://creativecommons.org/licenses/by/4.0/deed.es_ES.

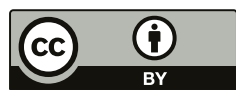

